\title{
Dysmegakaryopoiesis in myelodysplastic syndromes (MDS): An immunomorphometric study of bone marrow trephine biopsy specimens
}

\author{
J Thiele, $H$ Quitmann, S Wagner, R Fischer
}

\begin{abstract}
An immunohistochemical and morphometric analysis was performed on trephine biopsy specimens of the bone marrow in 40 patients (23 men and 17 women, mean age 62 years) with different subtypes of myelodysplastic syndromes (MDS) to determine dysmegakaryopoiesis, but particularly precursor cells-that is, pro- and megakaryoblasts. In 31 of the $\mathbf{4 0}$ patients the numbers of megakaryocytes were increased which was associated with a predominance of smaller cell forms (micromegakaryocytes). Compared with periodic acid Schiff, immunostaining with a formalin resistant monoclonal antibody against glycoprotein IHa (Y2/51(CD61) showed a clinically important proportion of immature elements. These could be designated pro- and megakaryoblasts by taking morphometric measurements on smears and bone marrow sections. There was a relevant increase in the number of promegakaryoblasts in 32 patients, consistent with uncontrolled expansion of the precursor pool. Seventeen repeated bone marrow biopsy specimens taken after karyopoiesis-that is, the occurrence of immature and small megakaryocytes with hypolobulated nuclei-has been described as the most typical feature. ${ }^{1-10}$ Moreover, in vitro cultures of haematopoietic progenitor cells derived from bone marrow in patients with $\mathrm{MDS}^{11-15}$ showed a striking defect of megakaryocyte colony formation. ${ }^{16}$ Although atypias of this cell lineage seem to have a major role in the evolution of this disorder, there is hardly any accurate information about the prevalence and size of megakaryocytes as well as pro- and megakaryoblasts. The introduction of a formalin resistant monoclonal antibody against glycoprotein (Gp) IIIa, termed Y2/51 (CD61), facilitates the evaluation of total megakaryocytopoiesis in routinely processed trephine biopsy specimens in addition to blood films and smears of marrow aspirates. ${ }^{1718}$ The combination of $\mathrm{Y} 2 / 51$ immunostaining and morphometry yields an unusual opportunity to determine the number and size of megakaryocyte precursors in bone marrow sections or in situ. ${ }^{19} 20$ In this paper we analysed dysmegakaryopoiesis in MDS by studying the trephine biopsy specimens of 40 patients and by immunomorphometrical methods.
\end{abstract} chemotherapy largely showed a decrease in the numbers of megakaryocytes including the precursor cell population. Moreover, morphometric evaluation disclosed that micromegakaryocytes in MDS differ significantly from those in chronic myeloid leukaemia (CML) due to distinctive nuclear features and a disturbed nuclear:cytoplasmic ratio. These changes generate a more pleomorphic or atypical appearance of this cell population in MDS, compared with micromegakaryocytes in CML.

It is concluded that the disproportionate increase in megakaryocyte precursors and the grossly abnormal aspects of micromegakaryocytes in MDS are characteristics of the severe defect

Institute of Pathology, University of Cologne, Joseph-Stelzmann Str. 9, D-500 Cologne 41, Germany J Thiele H Quitmann $S$ Wagner R Fischer

Correspondence to: Dr Juergen Thiele

Accepted for publication 13 November 1990 involving haematopoiesis in this disorder.

Myelodysplastic syndromes (MDS) include several morphological subtypes defined by cytological criteria. ${ }^{1}$ MDS may precede acute myeloid leukaemia (AML), sometimes by many years, and are further characterised by a clonal evolution, an ineffective and abnormal haematopoiesis, and variable degrees of cytopenias. $^{23}$ Among the various abnormalities affecting bone marrow morphology dysmega-

\section{Methods}

Accepted criteria for the definition of $\mathrm{MDS}^{23}$ were used in a total of 40 patients $(23$ men and 17 women, mean age 62 years). The classification of MDS according to the FAB criteria was as follows: refractory anaemia (RA) $(\mathbf{n}=$ 1); RA with ring sideroblasts (RARS) $(\mathbf{n}=2)$; RA with excess blasts (RAEB) $(\mathbf{n}=$ 27); chronic myelomonocytic leukaemia (CMML) $(n=8)$; RAEB in transformation (RAEB-T) $(\mathrm{n}=2) .{ }^{1}$ In 17 of the 40 patients enrolled in this study repeated bone marrow biopsies were performed at intervals ranging from two to 41 months and after chemotherapy. Of the 40 bone marrow specimens, 25 displayed a hyperplastic, 13 a normocellular, and two a hypoplastic subtype of MDS. Bone marrow samples from 15 patients (eight men and seven women, mean age 56 years) without any haematological disorders and a thrombocyte count within the normal range (150$\left.345 \times 10^{9} / 1\right)$ served as controls.

Trephine biopsy specimens of the bone marrow were taken from the posterior iliac crest $^{21}$ on admission and after chemotherapy. Fixation comprised aldehyde solution for 12 to 48 hours ( $2 \mathrm{ml} 25 \%$ glutardialdehyde, $3 \mathrm{ml}$ 
Figure 1A-F

Megakaryocytes in MDS after immunostaining with an antibody against glycoprotein IIIa (Y2/51 (CD61): comparison between smears of sternal aspirates (figs $1 A-C$ ) and sections of bone marrow tissue (figs $1 D-F$ ).

Smears: fig $1 \mathrm{~A}$ cluster of micromegakaryocytes; fig $1 B$ promegakaryoblast; fig $1 C$ megakaryoblast. Sections: fig $1 D$ micromegakaryocytes; fig $1 E$ promegakaryoblast (arrow head); fig $1 F$ megakaryoblast (arrow head).

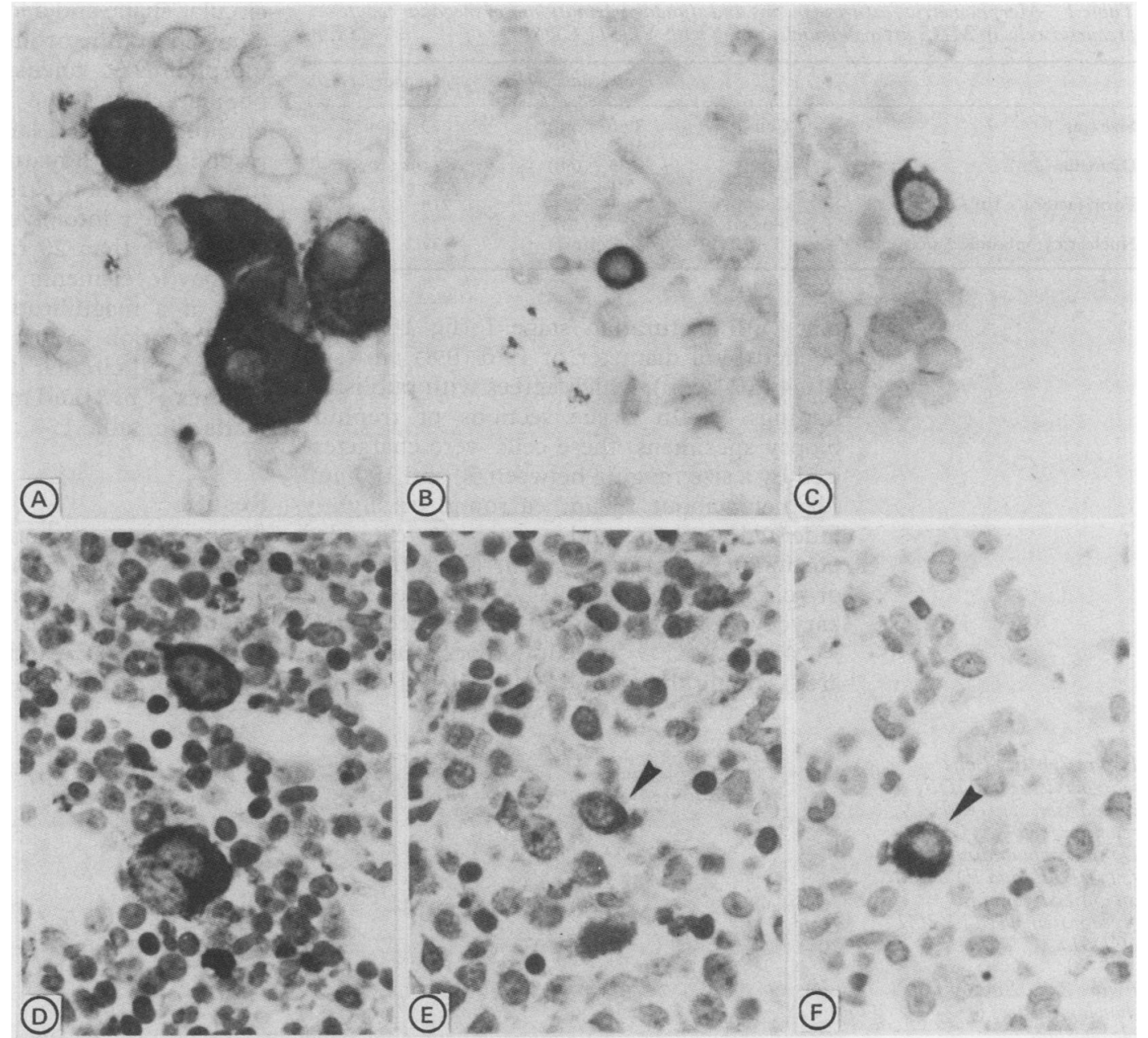

$37 \%$ formaldehyde, $1.58 \mathrm{~g}$ calcium acetate and distilled water per $100 \mathrm{ml}$ ), and further processing included decalcification for three to four days in $10 \%$ buffered EDTA, $\mathrm{pH} 7 \cdot 4$, paraffin wax embedding, and use of several staining methods. ${ }^{22}$

The monoclonal antibody Y2/51 (CD61), which is directed against Gp IIIa, was purchased from Dako-Diagnostica $\mathrm{GmbH}$ (Hamburg, Germany). Before staining, all slides of the paraffin wax embedded marrow specimens were predigested with pronase (1 $\mathrm{mg}$ per $1 \mathrm{ml}$ TRIS-buffered saline) for about 30 minutes at $37 \cdot 5^{\circ} \mathrm{C}$. Thereafter samples were stained using the alkaline phosphatase-anti-alkaline phosphatase (APAAP) technique, with neo-fuchsin as the alkaline phosphatase substrate. ${ }^{2324}$

Morphometric evaluation was performed using a manual optic pidnimeter (MOP-AMO1-Kontron) with a standard program set (Kontron software) on trephine biopsy specimens with an artefact-free marrow area of 6.9 (SD 2.5$) \mathrm{mm}^{2}$ after immunostaining with Y2/51 (CD61) and periodic acid Schiff (PAS).

Total count for megakaryocytes per square millimetre was obtained at $\times 500$ magnification by calculating the evaluable marrow area in the trephine biopsy specimen (excluding areas of haemorrhage and distortion as well as cortical and trabecular bone) and the total number of the corresponding PAS and glycoprotein IIIa (Y2/51) positive elements. In this way only nucleated megakaryocytes were sought-that is, no pyknotic-degenerative forms or naked (bare) nuclei-and no (anuclear) cytoplasmic fragments, to avoid erroneous counting of giant platelets and marginal sections of megakaryocytes.

As in previous studies ${ }^{1920}$ methods to characterise megakaryocyte precursors in smears and sections of bone marrow are only briefly described. For quantitative assessment of this cell population we performed a pilot study on smears of sternal aspirates stained with Y2/51 (CD61) from five patients. This preliminary investigation disclosed that, compared with the micromegakaryocytes of MDS (fig 1A), the most immature elements clearly recognisable as members of the megakaryocyte series-that is, promegakaryoblast ${ }^{25-27}$ had a diameter of $10.3(0.6) \mu \mathrm{m}$ (size $69.6(1.3)$ $\mu \mathrm{m}^{2}$ ) (fig 1B). In corresponding tissue sections, in addition to the micromegakaryocytes (fig $1 D)$, the most immature, glycoprotein IIIa (Y2/51) positive precursors (promegakaryoblasts) had a size of less than $50 \mu \mathrm{m},{ }^{2}$ a diameter about $8 \mu \mathrm{m}$, a round nucleus with dispersed chromatin, and a small rim of cytoplasm (fig 1E). On bone marrow smears there is a considerable flattening of megakaryocytes, so this decrease in size in tissue sections amounting up to $30 \%$ (spreading factor 1.3 to $1.5)$ is understandable. ${ }^{28}$ Accordingly, on smear preparations megakaryoblasts, consis- 
Table 1 Morphometric features (means and standard deviations) of megakaryocyte precursor cells in MDS after immunostaining with Y2/51 (CD61)

\begin{tabular}{llclcl}
\hline & & \multicolumn{2}{c}{ Promegakaryoblasts } & \multicolumn{2}{c}{ Megakaryoblasts } \\
\hline Size $\left(\mu \mathrm{m}^{2}\right)$ & Cell & $39 \cdot 6$ & $(3 \cdot 8)$ & $73 \cdot 4(4 \cdot 3)$ \\
Diameter $(\mu \mathrm{m})$ & Nucleus & $16 \cdot 2$ & $(2 \cdot 9)$ & $27 \cdot 2(3 \cdot 8)$ \\
& Cell & $7 \cdot 5(0 \cdot 8)$ & $11 \cdot 6(0 \cdot 8)$ \\
Form factor $\left(\times 10^{-3}\right)$ & Nucleus & $5 \cdot 1$ & $(0 \cdot 6)$ & $6 \cdot 6(0 \cdot 5)$ \\
Nuclear:cytoplasmic ratio $\left(\times 10^{-2}\right)$ & Nucleus & 751 & $(13)$ & 718 & $(5)$ \\
& & $40 \cdot 2$ & $(6 \cdot 7)$ & 737 & $(5)$ \\
& & & $37 \cdot 2$ & $(4 \cdot 8)$ \\
\hline
\end{tabular}
defined by a diameter of $19.6(0.8) \mu \mathrm{m}$ (size $\left.110.4(5.4) \mu \mathrm{m}^{2}\right)$, which agrees with published findings. ${ }^{26-29}$ In tissue sections of trephine biopsy specimens, these cells were characterised by a size ranging between 50 and $100 \mu \mathrm{m}^{2}$ (diameter about $13 \mu \mathrm{m}$ ), a round or slightly indented nucleus, and a not very extended portion of cytoplasm (fig $1 \mathrm{~F}$ ). The form factor or circular deviation (CD) of the megakaryocytes and their nuclei was defined as $C D$ $=4 \pi \mathrm{A} / \mathrm{C}^{2}(\mathrm{C}=$ circumference and $\mathrm{A}=$ area), giving the value $1000 \times 10^{-3}(1 \cdot 0)$ for a circular shape and a lower factor indicating an ellipsoid outline or increased irregularity. For morphometric calculation of this early subpopulation and the more mature, small to medium-sized and large megakaryocytes corresponding with maturation stages II to $I V,{ }^{29}$ the total area of the trephine biopsy specimens was divided into five segments of the same size. More than 20 randomly selected megakaryocytic elements were measured in each field at a magnification of 1250 times with determination of area, diameter, circumference and circular deviation. Morphometric features of pro- and megakaryoblasts are summarised in table 1 .

\section{Results}

An overview of bone marrow tissue from patients with MDS and stained by PAS shows that there was a remarkable clustering and predominance of pleomorphic small megakaryocytes (figs $2 \mathrm{~A}$ and $\mathrm{C}$ ). After immunostaining with Y2/51 (CD61) these features are most
Figure 2A-F Bone marrow tissue in $M D S$, comparison between $P A S$ reaction (figs $2 A, C$, and $D)$ and immunostaining (figs $2 B, E$, and $F$ ) with an antibody against glycoprotein IIIa (Y2/51 (CD61)

Figure $2 A$ Survey showing clusters of pleomorphic megakaryocytes.

Figure $2 B$

Immunostaining shows dislocation of very small to medium sized

megakaryocytes along the trabecular area (arrow heads).

Figure 2C Atypical micromegakaryocytes with hypolobulated dense nuclei and relatively small areas of cytoplasm.

Figures $2 D$ and $E$ Large megakaryocytes of the control group for comparison, displaying horseshoe-like nuclei surrounded by an extensive portion of cytoplasm.

Figure 2F Pleomorphic appearance of

megakaryocytes disclosed by immunostaining.

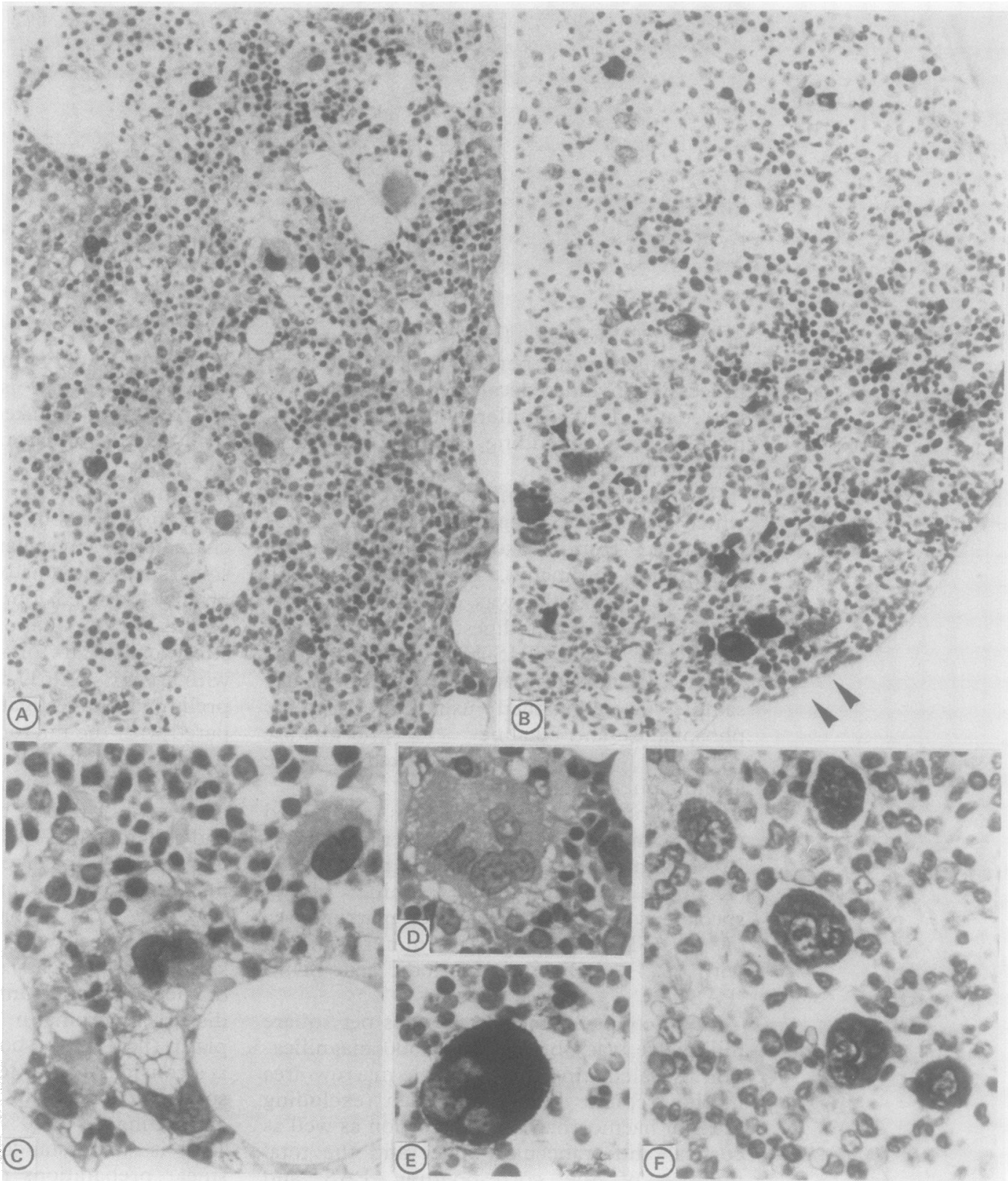


Table 2 Prevalence and size of megakaryocytes (mean and standard deviation) in MDS (normal values of control group are given in parentheses)

\begin{tabular}{lrlrl}
\hline Stain & \multicolumn{2}{c}{ Prevalence per $\mathrm{mm}^{2}$} & \multicolumn{2}{c}{ Size $\left(\mu \mathrm{m}^{2}\right)$} \\
\hline PAS & $30 \cdot 4$ & $(11 \cdot 1)$ & 297.4 & $(157.5)$ \\
& $(10 \cdot 6$ & $(3 \cdot 1))$ & $(338.6$ & $(47 \cdot 7))$ \\
Y2/51 (CD61) & 69.9 & $(38.3)$ & 226.0 & $(179 \cdot 7)$ \\
& $(22 \cdot 8$ & $(5 \cdot 5))$ & $(277.3$ & $(174.8))$ \\
\hline
\end{tabular}

prominently expressed and the appearance of micromegakaryocytes is conspicuous (figs $2 B$ and F). An atypical localisation at the endostal border, normally reserved for neutrophilic granulocytopoiesis, was often demonstrable (fig 2B). Compared with the megakaryocytes of the control group (figs $2 \mathrm{D}$ and $\mathrm{E}$ ), in MDS micromegakaryocytes show a relatively large, but hypolobulated nucleus surrounded by a not very extensive area of cytoplasm (fig 1D; figs 2C and F). Furthermore, immature elements (proand megakaryoblasts) are often disclosed (figs $1 \mathrm{E}$ and $\mathrm{F}$ ) which are not or only partially (such as megakaryoblasts) identifable by conventional staining methods. An increase in precursor cells as well as mature megakaryocytes (stages II to IV) ${ }^{29}$ was often, but not significantly, associated with an increase in reticulin (argyrophilic) fibres. Collagen fibrosis was not present.

In only nine of the 40 patients with MDS the numbers of megakaryocytes fell within the normal range, independent of bone marrow cellularity (hyper-, normo-, or hypocellular subtypes). The overall prevalence and size distribution of megakaryocytes are given in table 2. In this survey cases of MDS were compared with normal bone marrow specimens after a PAS reaction as well as immunostaining with Y2/51 (CD61). The differences were significant. Discordant measurements in size distribution are illustrated in fig 3. A pronounced left-shift, which results in a predominance of small, probably immature elements of the megakaryocytopoiesis after $\mathrm{Y} 2 / 51$ staining was evident. Detailed data on the prevalence of proand megakaryoblasts are given in table 3. Compared with normal bone marrow, a disproportionate expansion of the precursor pool of megakaryocytes (relative numbers) is common in many patients with MDS. In 25 of 40 patients an excess of the upper limit of normal for promegakaryoblasts was calculated, and correspondingly, for megakaryoblasts in 32 patients. Chemotherapy generated a significant decrease in the number of megakaryocytes and their precursor elements in 13 of 17 patients investigated (table 3 ).

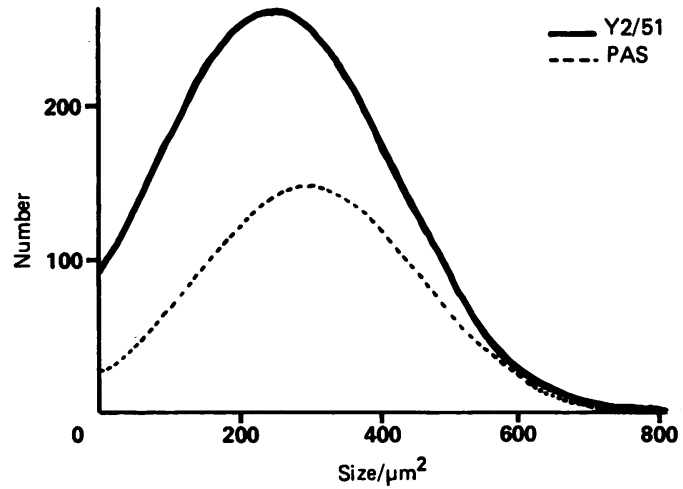

Figure 3 Size distribution of megakaryocytes in MDS after conventional $P A S$ reaction and immunostaining with a monoclonal antibody against glycoprotein IIIa (Y2/51 (CD61).

\section{Discussion}

Immunomorphological techniques were used to obtain an exact determination of megakaryocytopoiesis, thus extending the descriptive findings of cytological features in MDS. Moreover, in most patients an uncontrolled increase in pro- and megakaryoblasts was noticeable in bone marrow tissue compared with normal specimens. Dysmegakaryopoiesis was further characterised by the occurrence of abnormal micromegakaryocytes, highlighting obvious defects of maturation in comparison with CML specimens.

Exact quantitation of the numbers of megakaryocytes is only feasible by examining bone marrow biopsy specimens. ${ }^{30}$ For this reason studies based on smears which report a reduction in the numbers of cells of this lineage in $\mathrm{MDS}^{31}{ }^{32}$ should be treated with caution. Significant differences may be generated by methodology. ${ }^{6}$ Megakaryocytes are known to have a close affinity with marrow reticulin, and a varying degree of fibrosis is a common finding in MDS. ${ }^{6-10}{ }^{2}$ It is doubtful, therefore, whether aspirates yield a representative insight into the amount of these marrow elements. Furthermore, the numbers of megakaryocytes were related to bone marrow cellularity. ${ }^{32}$ This result was not possible from our study because only two cases had a hypocellular subtype of MDS. A recently published immunohistochemical study of bone marrow trephines in MDS which also used Y2/51 (CD61) showed a significant increase in numbers of megakaryocytes, confirming our findings. ${ }^{33}$

Previous studies on routinely stained smear preparations in patients with suspected "preleukaemia" showed a large number of micromegakaryocytes with a mean size of 618 (219)

Table 3 Morphometric features (mean and standard deviation) of megakaryocytopoiesis in MDS after immunostaining with Y2/51 (CD61)

\begin{tabular}{|c|c|c|c|c|c|c|}
\hline \multirow[b]{4}{*}{$\begin{array}{l}\text { Megakaryocytes (total per } \mathrm{mm}^{2} \text { ) } \\
\text { Promegakaryoblasts }\left(\text { per } \mathrm{mm}^{2} \text { ) }\right. \\
\text { Percent of total megakaryocytopoiesis } \\
\text { Megakaryoblasts (per } \mathrm{mm}^{2} \text { ) } \\
\text { Percent of total megakaryocytopoiesis }\end{array}$} & & \multicolumn{5}{|c|}{ Myelodysplastic syndromes } \\
\hline & & \multirow{2}{*}{\multicolumn{2}{|c|}{ Total }} & \multicolumn{3}{|c|}{ Sequential biopsies } \\
\hline & Control group & & & First trephine & \multicolumn{2}{|c|}{ Second trephine } \\
\hline & $\begin{array}{rr}22 \cdot 8 & (5 \cdot 5) \\
1.3 & (0.5) \\
5 \cdot 7 & (2 \cdot 1) \\
2 \cdot 1 & (0.8) \\
9 \cdot 4 & (3 \cdot 2)\end{array}$ & $\begin{array}{r}69 \cdot 9 \\
6 \cdot 3 \\
9 \cdot 1 \\
13 \cdot 5 \\
19 \cdot 2\end{array}$ & $\begin{array}{l}(38 \cdot 3) \\
(7 \cdot 2) \\
(6 \cdot 9) \\
(15 \cdot 3) \\
(12 \cdot 3)\end{array}$ & $\begin{aligned} 83 \cdot 4 & (46 \cdot 9) \\
9 \cdot 6 & (9 \cdot 1) \\
10 \cdot 0 & (6 \cdot 8) \\
22 \cdot 3 & (19 \cdot 1) \\
23 \cdot 7 & (12 \cdot 2)\end{aligned}$ & $\begin{array}{r}64 \cdot 0 \\
6 \cdot 6 \\
8 \cdot 8 \\
18 \cdot 2 \\
26 \cdot 9\end{array}$ & $\begin{array}{l}(31 \cdot 4) \\
(8 \cdot 1) \\
(9 \cdot 2) \\
(13 \cdot 7) \\
(15 \cdot 9)\end{array}$ \\
\hline
\end{tabular}


Table 4 Comparison of (micro)-megakaryocytes in CML and MDS with control group after immunostaining with Y2/51 (CD61) (mean and standard deviation)

\begin{tabular}{|c|c|c|c|c|c|c|}
\hline \multirow{2}{*}{$\begin{array}{l}\text { Cell } \\
\quad \text { Size }\left(\mu \mathrm{m}^{2}\right) \\
\quad \text { Form factor }\left(\times 10^{-3}\right)\end{array}$} & \multicolumn{2}{|c|}{$C M L(n=41)$} & \multicolumn{2}{|c|}{$M D S(n=40)$} & \multicolumn{2}{|c|}{ Controls ( $n=15$, } \\
\hline & $\begin{array}{l}193 \cdot 5 \\
787\end{array}$ & $\begin{array}{l}(122 \cdot 3) \\
(152)\end{array}$ & $\begin{array}{l}226 \cdot 0 \\
728\end{array}$ & $\begin{array}{l}(179 \cdot 7) \\
(143)\end{array}$ & $\begin{array}{l}277 \cdot 3 \\
778\end{array}$ & $\begin{array}{l}(174 \cdot 8) \\
(140)\end{array}$ \\
\hline $\begin{array}{l}\text { Nucleus } \\
\text { Size }\left(\mu \mathrm{m}^{2}\right) \\
\text { Form factor }\left(\times 10^{-3}\right) \\
\text { Nuclear:cytoplasmic } \\
\text { ratio }\left(\times 10^{-2}\right)\end{array}$ & $\begin{array}{l}42 \cdot 9 \\
574\end{array}$ & $\begin{array}{l}(30 \cdot 2) \\
(255)\end{array}$ & $\begin{array}{l}75 \cdot 2 \\
625\end{array}$ & $\begin{array}{l}(66 \cdot 3) \\
(202)\end{array}$ & $\begin{array}{c}77 \cdot 3 \\
589\end{array}$ & $\begin{array}{l}(55 \cdot 7) \\
(225)\end{array}$ \\
\hline
\end{tabular}

Data on CML are partially derived from a previous study. ${ }^{19}$ Only nucleated elements were sought-that is, excluding cytoplasmic fragments and naked (bare) and pyknotic nuclei.

$\mu \mathrm{m}^{2}{ }^{34}$ Provided that the spreading factor of about $30 \%{ }^{28}$ is taken into account in addition to the high standard deviation, this value falls in the range of measurements (bone marrow sections) reported in table 2. Qualitative abnormalities included in addition to normal sized megakaryocytes, a large number of immature and small cells with a compact or incompletely lobulated nucleus, and a hypogranulated cytoplasm-that is, micromegakaryocytes. ${ }^{14-10} 3334$ In the immunohistochemical study already referred to, ${ }^{33}$ all $\mathrm{Y} 2 / 51$ (CD61) positive "mononuclear cells" with a diameter of less than $15 \mu \mathrm{m}$ were regarded as micromegakaryocytes. This cell population comprised about $25 \%$ of the total megakaryocytopoiesis which was apparently evaluated by calculation of five high power fields per case $(n=11)$. As these determinations were entirely based on size (diameter) and not maturity, it is evident that this fraction included also pro- and megakaryoblasts with a diameter ranging between 7.5 to $11.6 \mu \mathrm{m}$ (table 1 ).

Because micromegakaryocytes are a prominent histopathological feature of CML, ${ }^{194-42}$ it is intriguing to compare this peculiar cell population with the small and pleomorphic megakaryocytes observed in MDS. In this context, immunostaining with Y2/51 (CD61) seems to be the most appropriate method of identifying all the elements of megakaryocytopoiesis. Morphometric characteristics of megakaryocytes in CML, which were partially derived from a previous study, ${ }^{19}$ permit such an evaluation. The results of this calculation are

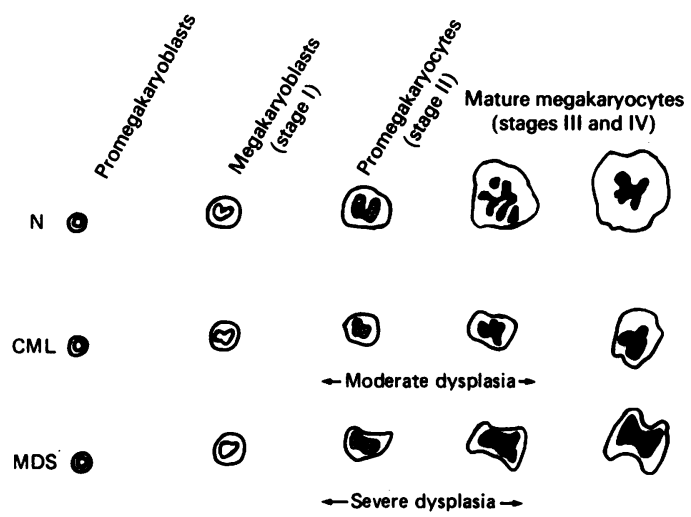

Figure 4 Histomorphometric characteristics of megakaryocyte maturation stages - that is, dysmegakaryopoiesis in $C M L$ and $M D S$ compared with the normal differentiation of a control group ${ }^{2629}$ shown by immunostaining with Y2/51 (CD61). summarised in table 4 and schematically illustrated in fig 4. Although there was no significant difference in size for both subtypes of micromegakaryocytes, in MDS a more irregular cell shape and a larger and more rounded nucleus were demonstrable, generating a greater deviation of the nuclear:cytoplasmic ratio (fig 4). These variables may account for the more abnormal-that is, dysplastic aspect-of the micromegakaryocytes in MDS compared with those of CML.

Analysis of dysplastic hematopoiesis in bone marrow cultures disclosed abnormal behaviour patterns, with common defects including absent, reduced, or even increased cluster formations of myeloid progenitor cells, ${ }^{11-14}$ but particularly after long term culture experiments. ${ }^{15}$ The ability megakaryocyte progenitors (CFU-M) have for colony formation is of special interest when considering the increase in promegakaryoblasts in many patients enrolled in this study (table 3 ). In a comprehensive investigation ${ }^{16}$ nine out of 10 patients with MDS displayed defective growth of CFU-M colonies, similar to findings for erythroid progenitor cells. ${ }^{12}$ It is tempting to speculate that this unruly expansion of the megakaryocyte precursor pool is indicative of a severe defect involving haematopoiesis in MDS which grossly impairs the normal regulation and maturation of this cell line..$^{27344}$

Supported by a grant from the Deutsche Forschungsgemeinschaft (DFG-Th 390/1-2).

1 Bennett JM, Catovsky D, Daniel MT, et al. Proposals for the classification of the myelodysplastic syndromes. $\mathrm{Br}$ Haematol 1982;51:189-99.

2 Jacobs A. Myelodysplastic syndromes: pathogenesis, functional abnormalities, and clinical implications. J Clin Pathol 1985;38:1201-17.

3 Tricot G, Mecucci C, Van den Berghe H. Evolution of the myelodysplastic syndromes. $\mathrm{Br} J$ Haematol 1986;63: 609-14.

4 Thiele J, Vykoupil K-F, Georgii A. Myeloid dysplasia (MD): a hematological disorder preceding acute and chronic myeloid leukemia. Virchows Arch (Pathol Anat) 1980;389:343-67.

5 Thiele J. Pathology of preleukemia. In: Lennert K, Hübner $\mathrm{K}$, eds. Pathology of the bone marrow. New York: $\mathrm{G}$ Fischer, 1984:70-86.

6 Tricot G, De Wolf-Peeters C, Hendriks B, Verwilghen RL. Bone marrow histology in myelodysplastic syndromes. I. Histological findings in myelodysplastic syndromes and comparison with bone marrow smears. Br J Haematol 1984;57:423-30.

7 Fohlmeister I, Fischer R, Mödder B, Rister M, Schaefer H-E. Aplastic anaemia and the hypocellular myelodysplastic syndrome: histomorphological, diagnostic, and prognostic features. J Clin Pathol 1985;38:1218-24.

8 Fohlmeister I, Fischer R, Schaefer H-E. Preleukemic myelodysplastic syndromes (MDS): pathogenetical considerations based on retrospective clinicomeril consequential studies. Anticancer Res 1985;5:179-88.

9 Frisch B, Bartl R. Bone marrow histology in myelodysplastic syndromes. Scand J Haematol 1986;36:21-37.

10 Delacretaz F, Schmidt P-M, Piguet D, Bachmann F, Costa J. Histopathology of myelodysplastic syndromes. The
FAB classification (proposals) applied to bone marrow FAB classification (proposals) applied to
biopsy. Am J Clin Pathol 1987;87:180-6.

11 Lidbeck J. In vitro colony and cluster growth in haemopoietic dysplasia (the preleukaemic syndrome) II. Identification of a maturation defect in agar cultures. Scand $J$ Haematol 1980;25:113-23.

12 Ruutu T, Partanen S, Lintula R, Teerenhovi L, Knuutila S. Erythroid and granulocyte-macrophage colony formation in myelodysplastic syndromes. Scand J Haematol 1984;32: 395-402.

13 Partanen $\dot{S}$, Juvonen E, Ruutu T. In vitro culture of haematopoietic progenitors in myelodysplastic syndromes. Scand J Haematol 1986;36(Suppl 45):98-101.

14 Dörmer $P$, Schalhorn A, Wilmanns W, Hershko C Erythroid and myeloid maturation patterns related to progenitor assessment in the myelodysplastic syndromes. Br J Haematol 1987;67:61-6.

15 Gebbia V, Miserendino V, Miceli S, et al. Analysis of human dysplastic haematopoiesis in long-term bone marrow culture. Blut 1989;59:442-8. 
16 Juvonen E, Partanen S, Knuutila S, Ruutu T. Megakaryocyte colony formation by bone marrow progenitors in myelodysplastic syndromes. $\mathrm{Br} J$ Haematol 1986;63: 331-4.

17 Erber WN, Jacobs A, Oscier DG, O'Hea AM, Mason DY. Circulating micromegakaryocytes in myelodysplasia. J Clin Pathol 1987;40:1349-52.

18 Gatter KC, Cordell JL, Turley $\mathrm{H}$, et al. The immunohistomorphological detection of platelets, megakaryocytes and thrombi in routinely processed specimens. Histopathology 1988;13:257-67.

19 Thiele J, Wagner S, Dienemann D, Wienhold S, Fischer R, Stein H. An immunohistomorphological study on megaStein $\mathrm{H}$. An immunohistomorphological study on megakaryocyte precursor cells in bone marrow tissue from patients with chronic myelc

20 Thiele J, Wagner S, Dienemann D, Wienhold S, Fischer R, Stein H. Megakaryocyte precursors (pro- and megakaryoblasts) within the normal human bone marrow. An immunohistochemical and morphometric study on routinely processed trephine biopsies. Analyt Quant Cytol Histol 1990;12:285-9.

21 Jamshidi K, Swaim JR. Bone marrow biopsy with unaltered architecture: a new biopsy device. J Lab Clin Med 1971;77:335-42.

22 Schaefer H-E. How to fix, decalcify and stain paraffin embedded bone marrow biopsies. In: Lennert $\mathrm{K}$, Hübner $\mathrm{K}$, eds. Pathology of the bone marrow. Stuttgart: $\mathrm{G}$ Fischer, K, eds. Parhe

23 Cordell JL, Falini B, Erber WN, et al. Immunoenzymatic labelling of monoclonal antibodies using immune complexes of alkaline phosphatase and monoclonal antialkaline phosphatase (APAAP complexes). J Histochem Cytochem 1984;32:219-29.

24 Stein H, Gatter KC, Asbahr H, Mason DY. Methods in laboratory investigation. Use of freeze-dried paraffinembedded sections for immunohistologic staining with monoclonal antibodies. Lab Invest 1985;52:676-83.

25 Vinci G, Tabilio A, Deschamps JF, et al. Immunological study of in-vitro maturation of

26 Breton-Gorius J, Vainchenker W. Expression of platelet proteins during the in-vitro and in-vivo differentiation of proteins during the in-vitro and in-vivo differentiation of
megakaryocytes and morphological aspects of their megakaryocytes and morphological asp

27 Mazur EM. Megakaryocytopoiesis and platelet production: a review. Exp Hematol 1987;15:340-50.

28 Levine RF, Hazzard KC, Lamberg JD. The significance of megakaryocyte size. Blood 1982;60:1122-31.

29 Williams N, Levine RF. The origin, development and regulation of megakaryocytes. $\mathrm{Br} J$ Haematol 1982;52: 173-80.
30 Singal R, Belliveau RR. Quantification of megakaryocytes in normal bone marrow. Analyt Quant Cytol Histol 1988;10: 33-6.

31 Vallespi T, Torrabadella M, Julia A, et al. Myelodysplastic syndromes: a study of 101 cases according to the FAB classification. Br J Haematol 1985;61:83-92.

32 Kitagawa M, Kamiyama R, Takemura T, Kasuga T. Bone marrow analysis of the myelodysplastic syndromes: histological and immunohistochemical features related to the evolution of overt leukemia. Virchows Archiv (Cell Pathol) 1989;57:47-53.

33 Fox SB, Lorenzen J, Heryet A, Jones M, Gatter KC, Mason DY. Megakaryocytes in myelodysplasia: an immunohistochemical study on bone marrow trephines. Histopathology 1990;17:69-74.

34 Wisneth M, Pflieger H, Kubaneck B, Heimpel H. Micromegakaryocytes in human bone marrow. Acta Haematol 1980;64:65-71.

35 Thiele J, Holgado S, Choritz H, Georgii A. Density distribution and size of megakaryocytes in inflammatory reactions of the bone marrow (myelitis) and chronic myeloproliferative diseases. Scand J Haematol 1983;89: 329-41.

36 Burkhardt R, Bartl R, Jäger $\mathrm{K}$, et al. Chronic myeloproliferative disorders (CMPD). Pathol Res Pract 1984; 1:131-86.

37 Georgii A, Vykoupil KF, Thiele J. Histopathology of bone marrow and clinical findings in chronic myeloproliferative disorders. In: Lennert K, Hübner K, eds. Pathology of the bone marrow. Stuttgart: G Fischer, 1984:147-69.

38 Frisch B, Bartl R, Burkhardt R, et al. Classification of myeloproliferative disorders by bone marrow histology. myeloproliferative disorders by

39 Frisch B, Lewis SM, Burkhardt R, Bartl R. Biopsy pathology of bone and bone marrow. London: Chapman and Hall, 1985.

40 Thiele J, Wienhold S, Zankovich R, Fischer R. A histomorphometric analysis of trephine biopsies of bone marrow from 65 patients with chronic myeloid leukemia. Classification of patients into subgroups with different survival patterns. Analyt Quant Cytol Histol 1990;12: 103-16.

41 Georgii A, Vykoupil KF, Buhr T, et al. Chronic myeloproliferative disorders in bone marrow biopsies. Path Res Pract 1990;186:3-27.

42 Burkhardt R, Jaeger K, Kettner G, Helmer G. Chronic myeloproliferative disorders: prognostic importance of myeloproliferative disorders: prognostic importance of

43 Hoffman R. Regulation of megakaryocytopoiesis. Blood 1989;74:1196-212.

44 McDonald TP. The regulation of megakaryocyte and platelet production. Int J Cell Cloning 1989;7:139-55. 